\title{
Hepatitis B Vaccination in Chronic Kidney Disease:- Experience in A Tertiary Care Centre
}

\author{
Dr Gomathy $S^{1}$, Dr Usha Samuel ${ }^{2}$, Dr Arun K A ${ }^{3}$, Dr Pradeep $B^{4}$ \\ ${ }^{1}$ Additional Professor, Dept Of Nephrology, Govt.T.D. Medical College, Alappuzha, India ) \\ 2(Professor Dept Of Nephrology, Govt.T.D. Medical College, Alappuzha, India) \\ 3 (Senior Resident Dept Of Nephrology, Govt.T.D. Medical College, Alappuzha, India) \\ 4(Senior Resident Dept Of Nephrology, Govt.T .D. Medical College, Alappuzha India)
}

\begin{abstract}
Background: The study was conducted to examine the seroconversion rate for hepatitis $B$ vaccination in Chronic Kidney Disease (CKD) patients and the factors which influence the sero conversion rate .

Methodology: This is a descriptive study which included 235 patients with CKD (chronic kidney disease) with $e G F R<60 \mathrm{ml} / \mathrm{min} / \mathrm{m}^{2}$ and they were given 40microgm of Hepatits B vaccine intramuscularly over deltoid at 0,1,2,6 months. Patient characteristics were recorded.Patient response was assessed by measuring antibodies to hepatitis $b$ surface antigen (AntiHBs Ab) six weeks after receiving the $4^{\text {th }}$ dose.

Results : 83\% of the patients were seroconverted with titre $\geq 10 I U / L$. The eGFR and and age are found to influence the titre. Aetiology of CKD and gender has no effect on the sero conversion status.

Conclusion: $H B V$ vaccination should be started to CKD patients at the earliest preferably in the first visit itselfas the seroconversion rate declines with progression of $C K D$.
\end{abstract}

Keywords: chronic kidney disease, hepatitis B vaccine,seroprotection

\section{Introduction}

Prevention of infectious disease is an important problem worldwide .Hepatitis B may be largely preventable by vaccination. Administration of HBVvaccination leads to the development of protective antibodies in responders. Patients with CKD especilay those on dialysis are at risk of contracting HBV infection ${ }^{(1)}$. Among HD patients susceptible to HBV infection the two year seroconversion HBV infection was $38.9 \%$ accounting for 19 seroconversions to HBsAg positivity per 100 patient years. The prevalence of HBV infection in Dialysis population in India ranges between $3.4-43 \%$ which is several folds higher than the carrier rate in general population $^{(2)}$.Strict procedures controlling transmission of blood borne infections during HD (hemodialysis) sessions as well as hepatitis B vaccinations are recommended and introduced on a mandatory basis in most HD centres world wide ${ }^{(1)}$. Although acute Hepatitis B infections tend to be mild and asymptomatic in dialysis patients ,up to two thirds may progress to chronic carriage, with subsequent risk of chronic liver disease, cirrhosis, liver cancer and nosocomial transmission within the HD unit and among the family members ${ }^{(3)}$.

The use of HBV vaccine and preventive measures ${ }^{(4)}$ have helped to reduce the annual incidence of HBV infection in patients on dialysis from 3\% to $0.05 \%$ between $1971-1997$ in USA. The usual vaccination schedule of HBV in adults with normal renal function is $10-20$ microgm/dose in a 3 shot schedule (at $0,1,6$ months).The effectiveness of HBV vaccination in ESRD (end stage renal disease) patients was found to be lower than in subjects without CKD. These patients commonly presented with lower seroconversion rates , lower peak antibody titres and a rapid decline in antibody levels. So they are given 40 microgm / dose in 4 shots $(0,1,2,6$ months).Early vaccination of patients before they start dialysis has been used in order to improve the response rate ${ }^{(2)}$.So $\mathrm{HBV}$ vaccination is recommended for all CKD patients and should be started in the first visit itself if possible after appropriate screening tests. Failure to complete the full course of HBV vaccination also impairs immunisation response ${ }^{(1)}$.

II.1 Aims and Objectives

\section{Materials And Methods}

1. To study the proportion of seroconversion after Hepatitis B vaccination in chronic kidney disease patients.

2. To study the factors affecting seroconversion.

II.2 Study Design

The study follows a descriptive design. 


\section{II.3 Inclusion Criteria}

All chronic kidney disease patients on conservative management with estimated GFR less than $60 \mathrm{ml} / \mathrm{min} / 1.73 \mathrm{~m}^{2}$ at the beginning of vaccination.

\section{II.4 Exclusion Criteria}

1. Patients on dialysis at the time of vaccination.

2. Patients who has not completed the required number of vaccinations or those who did not follow the recommended schedule or recommended dosage.

3. Patients on any immunosuppressive medications at the time of vaccinations.

4. Patients who did not give consent for the study.

\section{II.5 Methodology}

We collected data from 235 consecutive eligible patients who attended the outpatient department, Department of Nephrology Govt. T D Medical College Alappuzha, a tertiary care hospital in central Kerala, India during the study period from July 2015 to July 2016. The study was begun after obtaining approval from institutional research committee and ethics committee. All patients had undergone vaccination with recombinant hepatitis B vaccine $2 \mathrm{ml}$ (40microgram) intramuscularly in deltoid region with a total of 4 doses scheduled at 0,1 ,2, 6months. All patients underwent vaccination from our own hospital where potency of the vaccine is ensured before each dose. The study included patients who were vaccinated in the preceding three years. Anti HBS titre was done from a single laboratory between 6 weeks to 12 weeks after the last dose of vaccine using a sensitive assay where we used a cut off of $10 \mathrm{IU} / \mathrm{L}$.

The data collection was done over a period of one year (July 2015-June 2016).Data were collected from the medical records of patients and hospital records. Information regarding the etiology of CKD, serum creatinine at the first dose of vaccination, anti HBs antibody titre was collected along with the baseline characteristics of the patients. Estimated GFR was calculated using the Modified Diet in Renal Disease (MDRD) formula. Data analysis was done using spss version 16. Values are expressed as mean/median plus or minus standard deviations and as percentage for categorical parameters. Differences between groups are compared with Student's t-test for parametric continuous variables. Chi-square test is applied for estimating the occurrence of categorical variables. A P value $<0.05$ is used as the threshold of statistical significance.

\section{II.6 Ethical Clearance:}

The ethical approval was granted from the Institutional ethical Committee . Written informed consent was obtained from every patient prior to the examination

\section{Observations And Discussion \\ III. 1 Baseline Characteristics}

As part of the study information from 235 patients were collected and analyzed. The mean age of the population studied was 50.5 years with a standard deviation (SD) of 14.4 years. There were $64.3 \%$ males and $35.7 \%$ females (fig.1). The native kidney disease was divided into three broad categories . Glomerular disease, chronic tubulointestitial disease and others. Majority of the glomerular disease category was constituted by diabetic nephropathy, ischemic nephropathy, lupus nephritis and primary glomerular disease like $\operatorname{IgA}$ nephropathy etc. Patients grouped under the tubular disease include obstructive nephropathy, ADPKD ,etc. The proportion of each subgroup in our study is depicted in fig 2.

Figure1

\section{SEX DISTRIBUTION}

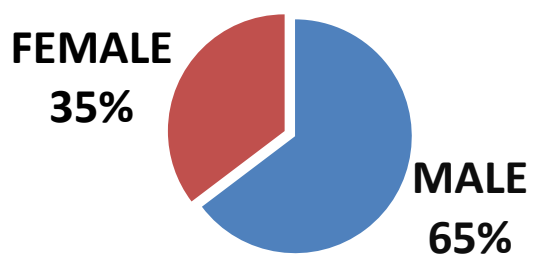


Figure 2

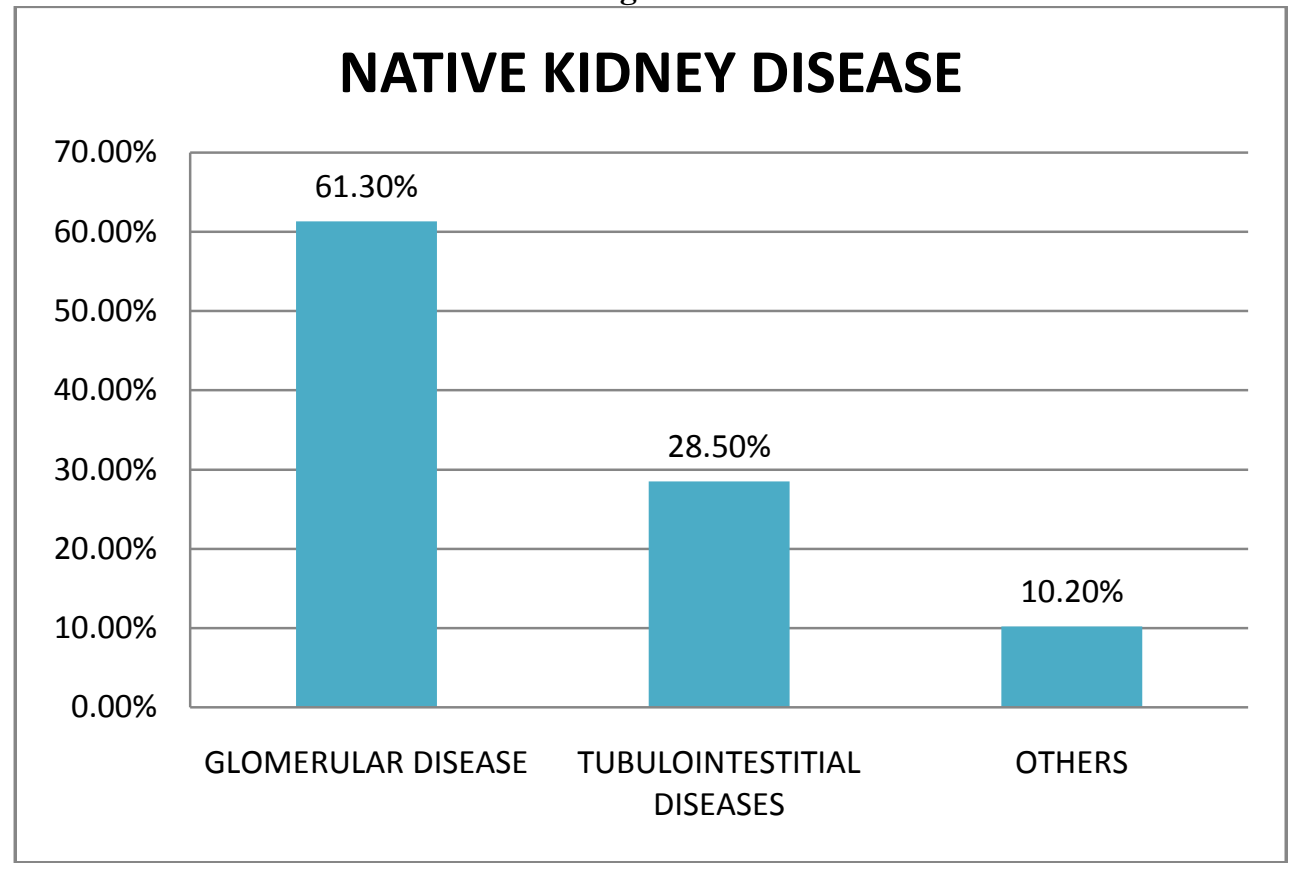

Unfortunately Hepatitis B vaccination of CKD patients is not routinely practiced in the periphery. So many patients reach our hospital unvaccinated even in end stage renal disease. In our cohort of patients also vaccination was carried out at different stages of CKD. $61.3 \%$ had their vaccination initiated when the serum creatinine was below $5 \mathrm{mg} / \mathrm{dl}$ but not less than $10 \%$ of subjects began their vaccination when the creatinine was well above $10 \mathrm{mg} / \mathrm{dl}$. This shown in fig 3.

Figure 3

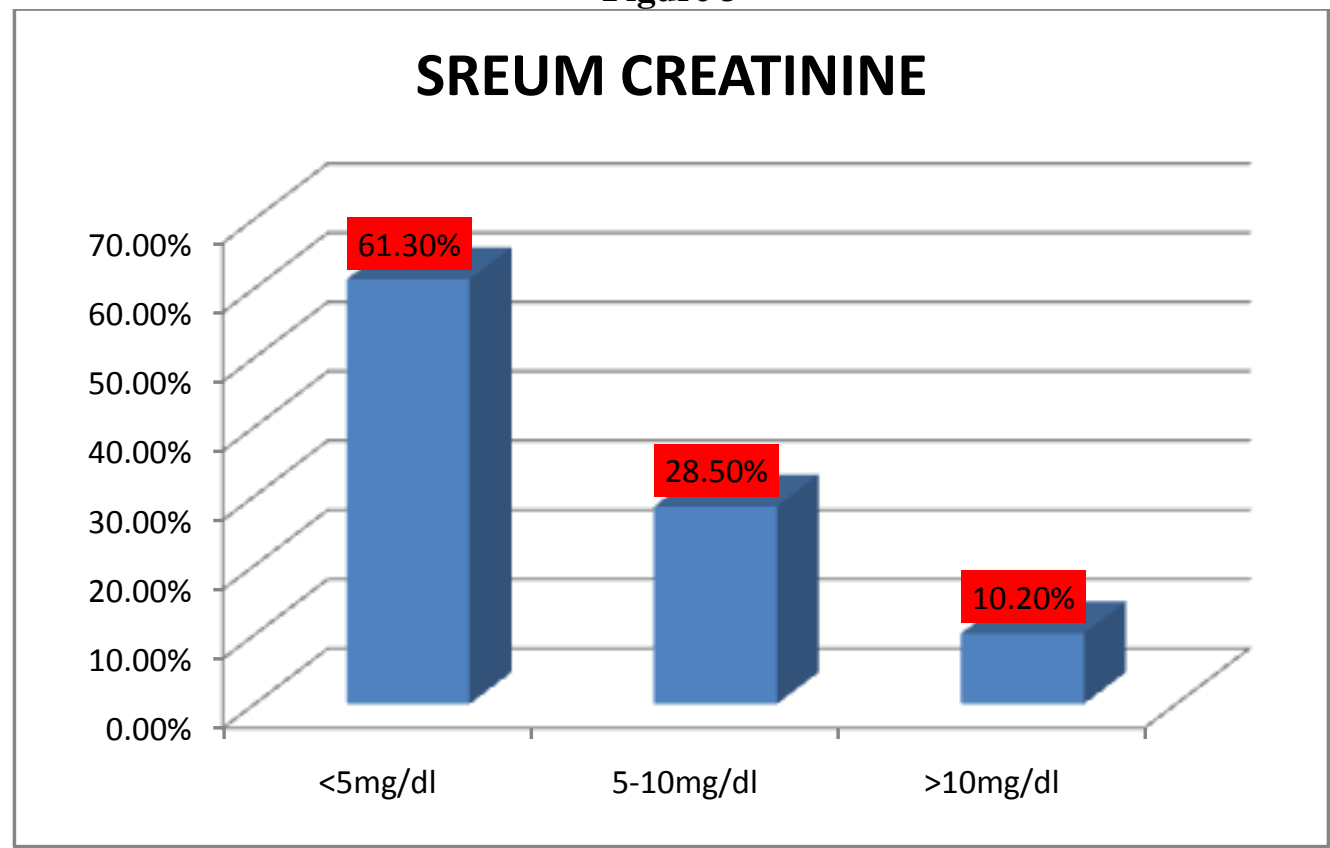

The mean serum creatinine at the initiation of vaccination is $4.69 \mathrm{mg} / \mathrm{dl}$ (SD $3.3 \mathrm{mg} / \mathrm{dl})$. Creatinine value ranged from 1-19mg/dl. Estimated GFR was calculated using the MDRD( Modified Diet in Renal Disease) formula. Patients were categorised into 4 based on the eGFR.( 44-60, 30-44,15-29, $\left.<15 \mathrm{ml} / \mathrm{min} / 1.73 \mathrm{~m}^{2}\right)$. eGFR ranged from $3-60$ with a mean of $26.29 \mathrm{ml} / \mathrm{min} / 1.73 \mathrm{~m}^{2}$ (SD-16.5).( fig 4 ) 
Figure 4

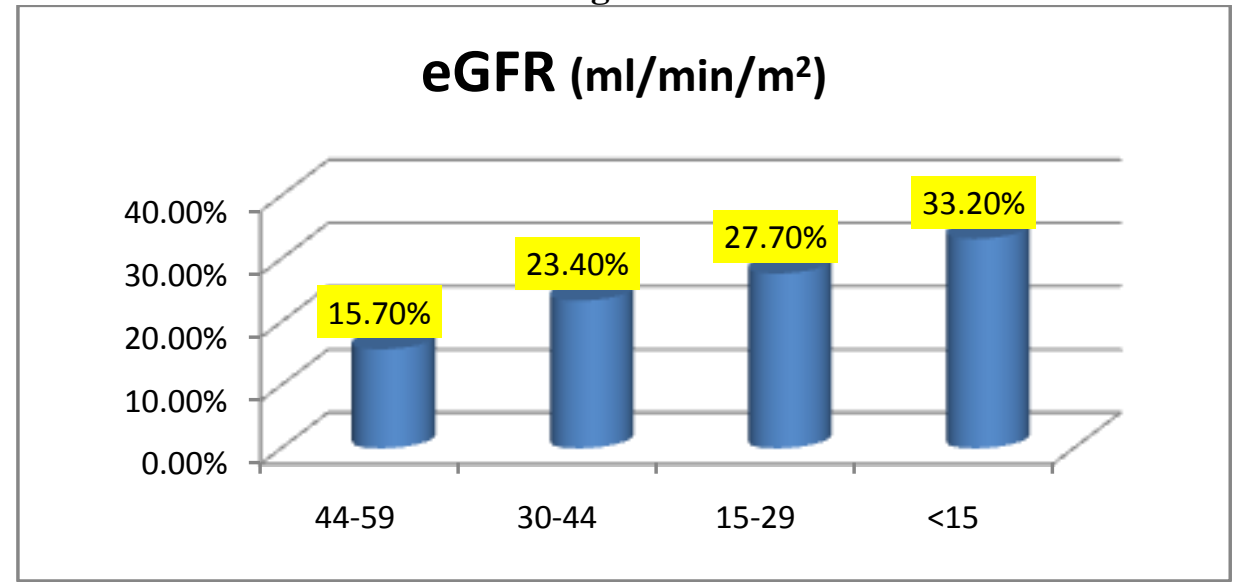

Figure 5

Anti HBS titre estimation is done 6 weeks after the last dose of vaccine. Out of the 235 patients 194 had titre more than $10 \mathrm{IU} / \mathrm{L}$. Maximum titre reported in our laboratory is $1000 \mathrm{IU} / \mathrm{L}$ and test has to be repeated after further dilution to know the exact titre above 1000 IU which is not done as it is laborious and do not seem to have any clinical relevance. $17.4 \%$ were reported to have inadequate titre (below $10 \mathrm{IU}$ ). These patients were initiated on repeat vaccination schedule. The titre did not follow normal distribution, still the mean titre was 297 IU. The curve has a positive skewing and around 55\% patients had their titre less than $200 \mathrm{IU} / \mathrm{ml} .12 \%$ subjects had strongly positive titres well above $1000 \mathrm{IU} / \mathrm{ml}$ which is clustered around the upper limit in the histogram given below (fig 6).

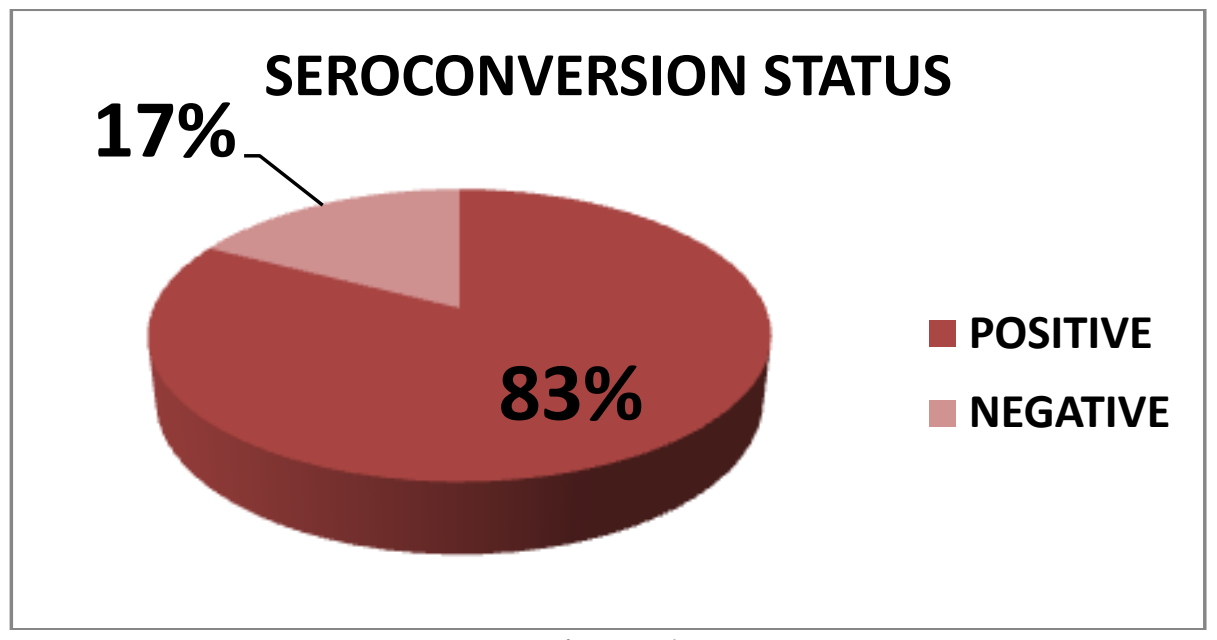

Figure 6

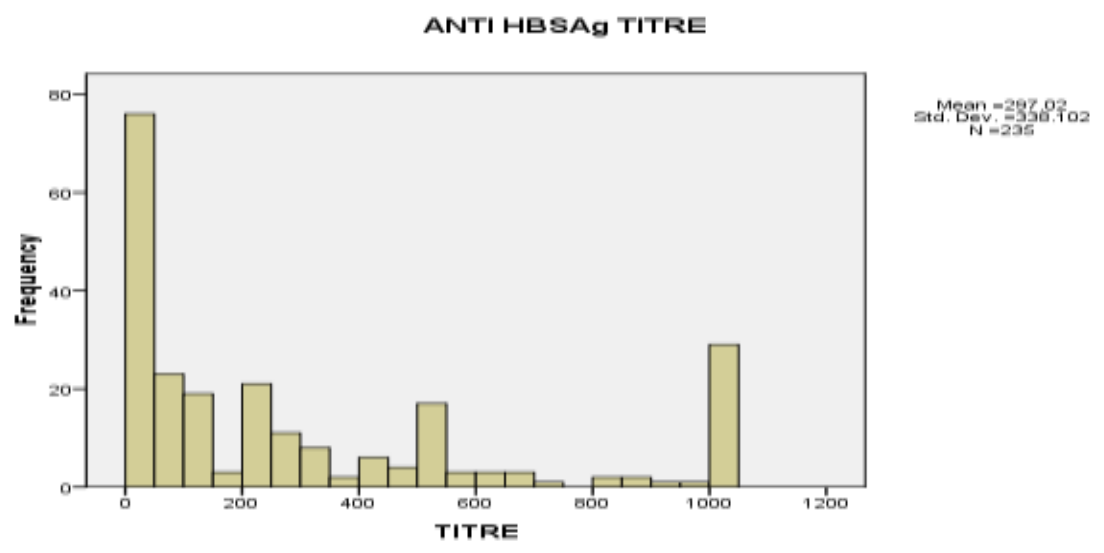


We have analyzed the factors that could affect the response to vaccination in CKD patients.

\section{II1.1 Age at the time of vaccination}

It is obvious that the rate of seroconversion declines as the age advances. Among our patients the mean age of patients with titre more than 10 IU was 48.2 and that of patients with titre less than 10 IU was 61.43 years . The difference is statistically significant (p-0.046).

\section{2 Gender}

We could not find any significant difference in seroconversion between male and female patients. (p-0.89) contrary to the earlier studies where male sex negatively correlated with seroconversion.

\section{3. Native Kidney Disease}

No statistically significant difference could be found among the three groups.

\section{III.4 . Estimated glomerular filtration rate}

The difference among the eGFR subgroups were found to be statistically significant. (p-<0.005). The titre positive group had a mean GFR of $27.47 \mathrm{ml} / \mathrm{min} / \mathrm{m}^{2}$ against $19.83 \mathrm{ml} / \mathrm{min} / \mathrm{m}^{2}$ in the titre negative patients.

We also did correlation analysis and found that there is positive correlation between GFR and anti HBStitre and negative correlation between age Vs GFR and serum creatinine Vs anti HBS titre.

\section{IV .Conclusions}

Hepatitis B vaccination in CKD patients with GFR less than $60 \mathrm{ml} / \mathrm{min} / \mathrm{m}^{2}$ has a failure rate of $17 \%$. Our study also shows that the anti HBS titre decreases as the age of the patient at the time of vaccination increases. Similarly antiHBS titre is low when the eGFR is low at the time of vaccination. The etiology of kidney disease or gender has no effect on the response to vaccination contrary to the literature which shows that male gender has impaired seroconversion.

Hepatitis B virus (HBV) infection is a serious global health problem. The prevalence of viral hepatitis B is higher in dialysis patients than in the general population because of the opportunity for exposure during the dialysis procedure. Immunization is the most effective way to prevent transmission of hepatitis B virus (HBV) and hence the development of acute or chronic hepatitis B. It is well established that patients with end-stage renal disease including dialysis-dependent patients, have an impaired immune response to hepatitis B vaccine. End stage renal diseases (ESRD) patients have lower seroconversion rates compared with the subjects with intact renal function. Moreover, even after the completion of vaccination schedule anti-hepatitis B (anti-HBs) titers of responder dialysis patients are low and decline logarithmically with time.The impaired efficacy of HBV vaccine in patients with ESRD has been attributed to numerous factors such as immune compromise because of uremia and some other factors.

Less severe immune abnormalities in early CKD stages give a chance for higher seroconversion rates, If patients are vaccinated early, pre-dialysis HBV infection may be avoided,

A response to booster doses in patients responding for the primary vaccination in early CKD stages is usually maintained during RRT. Hence it is highly essential to screen the vaccinated individuals periodically for the presence of optimal antiHBs titre and if shows decline in its level, may be given booster doses.

\section{Suggestions}

The study undoubtedly shows that the response to vaccination and titre is low in advanced CKD patients. We could also demonstrate the habit of delayed initiataion of vaccination in the CKD patients. The result gives a strong evidence to recommend initiation of vaccination at the earliest in CKD patients. Eventhough we don't have direct evidence, it is advisable to vaccinate patients who are at risk of developing CKD as theoretically immune response to vaccination fades with establishment of chronic kidney disease.CKD is asymptomatic especially in its early phase and at which time these patients are taken care of by the primary care physicians especialy in developing countries because of the dearth of renal care physicians. So patients at risk of developing CKD (those Diabetes Mellitus, Hypertension, urolithiasis, various systemic diseases ,family history of CKD, Chronic NSAID ingestion etc ) should be screened and if features of CKD are evident should be given the Hepatitis B vaccination at the earliest after appropriate screening tests. So it is highly essential to create an awareness among primary care physicians in this regard especially in developing countries . 


\section{VI .Limitatins}

1. The study lacks a control group to compare the response to vaccination. It would have been ideal to have another cohort without CKD or CKD with GFR $>60 \mathrm{ml} / \mathrm{min} / \mathrm{m}^{2}$ for comparison as that would have provided more useful information and eliminated the role of any possible confounding factors in the study although ample data are available in the literature for comparison.

2. The study considered the aetiology of renal failure as broad categories and hence the effect of each etiology on vaccination could not be inferred.

4. There are many issues unaddressed in this study like effect of dialysis, fate of non responders to repeat vaccination, need for routine booster dosing etc.A long term follow up study with serial measurement of titre is needed to solve these unresolved queries.

\section{References}

[1]. Alicja E. Grzegorzewska Hepatitis B vaccination in Chronic kidney disease : Review of evidence in non dialysed patients Hepat Mon.2012;12(11):e7359

[2]. Shireen Siddiqui1, Abida Malik1, Indu Shukla1, Meher Rizvi1, Shahzad F. Haque2

[3]. Seroprotection after hepatitis B vaccination in chronic kidney disease patients with modified schedule and dosage India ; J Infect Dev Ctries 2010; 4(6):389-392.

[4]. Mohemmed Hussein Somi, Babak Hajipour.Improving Hepatitis B Vaccine efficacy in Endstage renal disease patients and the role of Adjuants (Review article )International scholarly Research Network ,ISRN Gastroenterology; volume 2012,Article ID960413,9pages Doi:10.5402/2012/960413

[5]. Nicolas Janus, Launay-Vincent Vacher, Svetlana Karie, Elena Ledneva and Gilbert Deray Vaccination and Chronic Kidney Disease, Nephrol Dial Transplant (2008) 23: 800-807

[6]. Guidelines for vaccinating kidney dialysis patients and patients with CKD :Recommendations from Advisory Committee on Immunisation Practices (ACIP) CDC(December 2012) 